\title{
Paricalcitol reverses metabolic syndrome-associated heart failure through enhanced mitochondrial fusion
}

\author{
Hina Lateef Nizami ${ }^{1}$, Parmeshwar Katare ${ }^{1}$, Pankaj Prabhakar ${ }^{2}$, Ramu Adela ${ }^{2}$, Soumalya \\ Sarkar $^{1}$, Sudheer Arava ${ }^{2}$, Praloy Chakraborty ${ }^{3}$, Subir Maulik ${ }^{2}$, and Sanjay Banerjee ${ }^{1}$ \\ ${ }^{1}$ Translational Health Science and Technology Institute \\ ${ }^{2}$ All India Institute of Medical Sciences \\ ${ }^{3}$ Vardhman Mahavir Medical College and Safdarjung Hospital
}

June 8, 2020

\begin{abstract}
Background \& Purpose: We have earlier shown that vitamin D deficiency induces heart failure, in part, through insulin resistance. Whether activation of vitamin D receptor can attenuate heart failure, associated with metabolic syndrome, requires investigation. We aimed to assess cardioprotective potential of paricalcitol, a vitamin D receptor-activator, in high fat high fructose-fed rats. Experimental approach: Male Sprague Dawley rats were fed with control (Con) or high fat high fructose (HFHFrD) diet for 20 weeks. After 12 weeks, rats from HFHFrD group were divided into: HFHFrD, HFHFrD $+\mathrm{P}$ (paricalcitol i.p. $0.08 \mathrm{ug} / \mathrm{kg} /$ day) and HFHFrD+E (enalapril maleate i.p. $10 \mathrm{mg} / \mathrm{kg} /$ day). Intraperitoneal glucose tolerance test, blood pressure measurement and 2D echocardiography were performed. Cardiac fibrosis was assessed in Masson trichrome-stained paraffin-embedded heart sections. Mitochondrial DNA and proteins, and citrate synthase activity were measured in rat hearts. VDR was silenced in $\mathrm{H} 9 \mathrm{c} 2$ cardiomyoblasts and immunoblotting was performed. Key Results: Paricalcitol improved glucose tolerance, serum lipid profile and blood pressure in high fat high fructose-fed rats. Paricalcitol reduced cardiac wall thickness, and increased ejection fraction in high fat high fructose-fed rats, with no effect on perivascular fibrosis. PGC1- $\alpha$ was uprgulated in HFHFrD +P group compared to HFHFrD group, without significant difference in mitochondrial content. Citrate synthase activity was significantly higher in HFHFrD $+\mathrm{P}$ group compared to HFHFrD group. Rat hearts of HFHFrD $+\mathrm{P}$ group had significantly higher expression of mitofusins. H9c2 cells with VDR knockdown showed significantly lower expression of Mfn2. Conclusion \& Implications: Paricalcitol is cardioprotective in rats with metabolic syndrome, through improved mitochondrial dynamics, indicating repurposing potential.
\end{abstract}

\section{INTRODUCTION}

Heart failure is a public health problem with persistently rising prevalence due to increase in ageing population (1). Maladaptive cardiac remodeling, in response to injury or comorbid conditions such as diabetes and hypertension, and ventricular dilatation and impaired contractility constitute systolic heart failure (2). Its diagnosis is clinically confirmed by echocardiographic observation of impaired myocardial and valvular structure and function. Treatment options include lifestyle changes, pharmacologic therapy, implantable devices and surgery. Drug therapy is mainly aimed at relief of symptoms (e.g. diuretics) and improvement of residual cardiac function (e.g. ACE inhibitors, beta blockers and aldosterone antagonists). Heart failure often coexists with other morbidities such as metabolic syndrome, renal impairment and anemia, which complicates the treatment further.

We have earlier shown vitamin D deficiency is associated with increased prevalence of type 2 diabetes with and without coexisting coronary artery disease, both important risk factors for heart failure (3). Conditions such as chronic renal failure precipitate vitamin D deficiency as well as cardiac failure (4). In experimental 
models of vitamin D receptor (VDR) knockdown or diet-induced vitamin D deficiency, remarkable cardiac hypertrophy, activation of the renin angiotensin system and cardiac dysfunction are observed (5-6). However, experimental as well as clinical reports of vitamin D supplementation yield contradicting data about possible benefits (7-8). Reduced activation of vitamin D due to hepatic or renal dysfunction, and sequestering of vitamin $\mathrm{D}$ in fat stores in obesity are some possible reasons for loss of beneficial effects in disease, and hence synthetic, non-hypercalcemic analogues might be preferable alternatives (9-10). Paricalcitol is an FDAapproved drug used in the management of end-stage kidney disease, and has shown cardiorenal benefits in various experimental models (11-12).

Though heart failure is essentially a decompensated state of cardiac muscle contractility, it is also marked by deficits in cardiac bioenergetics. Energy transduction in the heart depends majorly on oxidative phosphorylation in the mitochondria; excessive fatty acid oxidation in the absence of glucose leads to respiratory uncoupling and oxidative stress (13). Mitochondrial dynamics are under control of processes such as fusion, fission and biogenesis, and insulin is known to directly promote mitochondrial fusion through optic atrophy protein 1 (OPA1) (14). Myocardial insulin resistance and mitochondrial dysfunction have been shown to be involved in the transition of heart from diastolic dysfunction to systolic failure in a model of pressure overload-hypertrophy in rats (15). Successful use of therapies such as trimetazidine, omega-3 fatty acid and perhexiline indicates that metabolic modulation might be an attractive therapeutic strategy to manage the development and progression of heart failure (16-17).

Vitamin D receptor is a nuclear receptor conventionally known to be involved in transcription of target genes involved in calcium absorption and bone formation. However, the pleiotropic extraskeletal effects of VDR are under investigation in various tissues (18-19). Vitamin D has been shown to favourably affect metabolic functions such as insulin signalling and lipid metabolism in both experimental and clinical samples (20-22). Regulation of mitochondrial structure, content and bioenergetics by 1,25-OH vitamin D3 has been reported in peripheral blood mononuclear cells, adipocytes, skeletal muscle and various cancer cell lines (2326). In homocysteine-treated rat heart slices, treatment with vitamin D3 improved mitochondrial function and redox status (27). However, the possible effect of VDR modulation on mitochondrial dynamics in heart, and intervention with vitamin D analogues in metabolic syndrome-associated cardiac failure requires investigation.

We aimed to investigate the therapeutic potential of paricalcitol, a known VDR agonist in cardiac dysfunction in rats with metabolic syndrome. Animals were fed with high fat high fructose diet, and treated with paricalcitol, and cardiometabolic outcomes were assessed. Since renin angiotensin system blockade has known clinical benefit against cardiac remodeling and failure, enalapril was used as standard therapy in a group of high fat high fructose fed rats.

\section{RESEARCH DESIGNS AND METHODS}

\section{Animals}

Male Sprague Dawley rats (200-220 gm) were procured from All India Institute of Medical Sciences, New Delhi and maintained in Small Animal facility of THSTI, Faridabad. Rats were provided food and water ad libitum throughout the study period, with the exception of the 12-hour fasting preceding blood sample withdrawal and intraperitoneal glucose tolerance test. All experimental procedures involving animals were performed according to the relevant regulations and guidelines of Institutional Animal Ethical Committee (IAEC) of Translational Health Science and Technology Institute, Faridabad (IAEC/THSTI/2015-3.

\section{Materials}

Paricalcitol was purchased from Cayman Chemical Company (\#17716). Enalapril maleate was procured from LKT Labs (\#E5201). Antibodies against VDR (ab3508), PGC1- $\alpha$ (ab54481), MFN1 (ab57602), and MFN2 (ab137037) were purchased from Abcam. Primers used in PCR-based estimation of mitochondrial content are listed in Table 1.

\section{In Vivo Study Design and Drug Treatment}


Control (65\% corn starch diet; Cat. No. D11708B) and high-fat high-fructose (45\% kcal\% fat and 35\% kcal\% fructose; Cat. No. D08040105G) diets were purchased from Research Diets, USA. After an acclimatization period of one week, rats were randomised into two groups, and were fed with either control diet (CON) or high-fat high-fructose diet (HFHFrD) for 20 weeks. At the end of 12 weeks, intraperitoneal glucose tolerance test and echocardiography were performed, and rats from HFHFrD group were further divided randomly into three groups: HFHFrD, HFHFrD+P (paricalcitol i.p. $0.08 \mathrm{ug} / \mathrm{kg} /$ day) and HFHFrD $+\mathrm{E}$ (enalapril maleate i.p. $10 \mathrm{mg} / \mathrm{kg} /$ day) (Figure 1). At the end of remaining 8 weeks of treatment, rats from all the groups were sacrificed; serum samples and heart tissues were collected and stored at $-80^{\circ} \mathrm{C}$ refrigerator for further analysis.

\section{Intraperitoneal Glucose Tolerance Test}

At the end of 12 and 20 weeks, after an overnight fasting period, rats were given a bolus injection of glucose ( $2 \mathrm{~g} / \mathrm{kg}$ I.P.). Blood glucose sampling was performed at 0 (just before), 15, 30, 60 and 120 min post glucose administration, using a commercially available blood glucose measurement system (OneTouch Select Plus, Johnson \& Johnson).

\section{Serum Biochemistry}

Insulin (Crystal Chem, USA), triglycerides (BioVision, USA), HDL cholesterol (BioVision, USA) and free fatty acids (BioVision, USA) were measured in rat serum samples using commercially available assay kits.

\section{Non Invasive Blood Pressure Measurement}

Blood pressure was evaluated at the end of the study (20 weeks) using the Non Invasive Blood Pressure System (Harvard Apparatus) complying with manufacturer's protocol. Animals were trained daily in restrainers for 4 days prior to experimental data recording. To record blood pressure, tail cuff and sensor were placed about $2 \mathrm{~cm}$ from the tip of the animal's tail, and blood pressure was recorded. Five individual measurements were made and analysed as mean for each animal.

\section{Echocardiography}

Echocardiography was performed after the completion of 20 weeks. Rats were anesthetized using a cocktail of ketamine $(80 \mathrm{mg} / \mathrm{kg} \mathrm{BW}$ i.p. $)$ and xylazine $(8 \mathrm{mg} / \mathrm{kg}$ BW i.p. $)$. Two-dimensional and M-mode echocardiograms were obtained using a using a fully digitized instrument (HD11 XE, Philips) with a hand-held $12 \mathrm{MHz}$ neonatal cardiac probe transducer placed at a short axis view at the level of the papillary muscles of the left ventricle. Diastolic left ventricular posterior wall (LVPW) and intraventricular septum (IVS) thicknesses were recorded. Left ventricular internal diastolic dimension (LVIDd) and LV internal systolic dimension (LVIDs) were also recorded. Fractional shortening and ejection fraction values were derived from the dimensions recorded above. Image acquisition and analysis was performed by an analyst blind to the experimental groups.

\section{Cardiac hypertrophy measurement}

Heart weight to-tail length ratio of rats was used to evaluate cardiac hypertrophy. Briefly, at the end of 20 weeks, animals from all groups were sacrificed, hearts were isolated, rinsed in ice-cold PBS and blotted, and their weight was measured. Ratio of heart weight and tail length was calculated and expressed in $\mathrm{gcm}^{-1}$.

\section{Histopathology}

At the end of 20 weeks, heart tissue was harvested and fixed in phosphate-buffered formalin (10\%). Fixed samples were embedded in paraffin and sectioned for histopathological analysis. Sections of 5 - $\mu \mathrm{m}$ thickness were stained with hematoxylin and eosin and Masson's trichrome, and examined under a light microscope.

\section{Cell Culture}

$\mathrm{H} 9 \mathrm{c} 2$ cell line was procured from ATCC (USA), and cultured in DMEM with $10 \% \mathrm{FBS}$, in a $5 \% \mathrm{CO}_{2}$ incubator at $37^{\circ} \mathrm{C}$. VDR in $\mathrm{H} 9 \mathrm{c} 2$ cells was silenced using shRNA (Origene) transfected using Xtremgene 
HP Transfection Reagent (Roche). Successfully transfected cells were selected using puromicin and used to establish a stable cell line.

\section{Western Blotting}

Rat heart tissues or H9c2 cells were lysed in ice-cold RIPA buffer (Pierce), and were then centrifuged at $12000 \mathrm{rpm}$ for 20 minutes at $4^{\circ} \mathrm{C}$. Bicinchonic acid assay (Thermo Scientific) was used to measure protein content. Proteins $(30 \mu \mathrm{g})$ were resolved on 10-12\% SDS-polyacrylamide gel using TGX stain free kit (BioRad). After electrophoresis, proteins were blotted on to polyvinylidine difluoride (PVDF) membrane (Merck Millipore). Membrane was blocked in 3\% non-fat dry milk in TBST (0.1\% Tween 20) or 5\% BSA solution at room temperature for an hour, followed by incubation with desired primary antibody overnight at $4^{\circ} \mathrm{C}$. After three washes with TBST, membrane was incubated with appropriate HRP-labeled secondary antibody at room temperature for an hour. Membrane was washed with TBST (thrice for 5 min each) and the blot was visualized using Gel Doc XR system (Bio-Rad), using Roche Lumi Light substrates (Thermo Scientific). Protein expression was normalised to corresponding stain free gel (BioRad ${ }^{\mathrm{TM}}$ ) loading controls' expression.

\section{RESULTS}

\section{Paricalcitol improves glucose tolerance in high fat high fructose-fed rats}

Fasting blood glucose and serum insulin

There was a significant increase in fasting blood glucose level of HFHFrD group as compared to the Con group, at the end of 20 weeks (Fig. 3.3A). However, we did not observe any significant difference in HFHFrD $+\mathrm{P}$ and HFHFrD + E groups, in comparison to HFHFrD group (Fig. 2A).

Similarly, there was a significant increase in fasting serum insulin level of HFHFrD group as compared to the Con group (Fig. 2B). However, no significant difference was observed in HFHFrD $+\mathrm{P}$ and $\mathrm{HFHFrD}+\mathrm{E}$ groups, in comparison to HFHFrD group (Fig. 2B).

HOMA analysis

At the end of twenty weeks, HOMA-IR of HFHFrD group was significantly higher than that of Con group (Fig. $2 \mathrm{C}$ ). However, no significant difference was observed in HFHFrD $+\mathrm{P}$ and $\mathrm{HFHFrD}+\mathrm{E}$ groups, in comparison to HFHFrD group (Fig. 2C).

No significant difference was found between HOMA-B values of different groups (Fig. 2D).

Intraperitoneal glucose tolerance test

In the IPGTT performed at the end of 12 weeks, HFHFrD group showed raised blood glucose time profile and significantly higher area under curve (AUC) than the Con group (Fig. 2E), indicating impaired blood glucose disposal in these rats.

In the IPGTT performed at the end of 20 weeks, HFHFrD group showed impaired glucose tolerance and higher AUC, compared to Con group (Fig. 2F-G). Upon 8 weeks of treatment with paricalcitol, HFHFrD $+\mathrm{P}$ group showed significantly improved glucose tolerance in IPGTT, with significantly lower area under curve, compared to HFHFrD group (Fig. 2F-G). The improvement of insulin resistance obtained after paricalcitol tretament is similar to the tretament of standard drug, enalapril.

\section{Paricalcitol attenuates dyslipdemia in high fat high fructose-fed rats}

We observed significantly higher serum triglycerides and free fatty acids in the HFHFrD group compared to Con group (Fig. 3A-B). Upon 8 weeks of treatment with paricalcitol, HFHFrD+P group showed significantly decreased triglycerides and free fatty acids, compared to HFHFrD group (Fig. 3A-B). There were no significant changes in serum HDL cholesterol in the different groups (Fig. 3C).

Paricalcitol attenuates hypertension in high fat high fructose-fed rats 
There were significant increases in systolic, diastolic and mean arterial blood pressure in HFHFrD group, compared to Con group (Fig. 3D-F). Upon 8 weeks of treatment with paricalcitol or enalapril, HFHFrD $+\mathrm{P}$ group showed significantly decreased blood pressure, compared to HFHFrD group (Fig. 3D-F).

\section{Paricalcitol attenuates cardiac hypertrophy in high fat high fructose-fed rats}

At the end of 20 weeks, we observed significant increase in the heart weight-to-tail length ratio in HFHFrD group, when compared to Con group (Fig. 3G). After treatment with paricalcitol or enalapril for 8 weeks, both $\mathrm{HFHFrD}+\mathrm{P}$ and $\mathrm{HFHFrD}+\mathrm{E}$ groups showed significantly decreased heart weight-to-tail length, as compared to HFHFrD group (Fig. 3G).

Paricalcitol attenuates left ventricular hypertrophy and systolic dysfunction in high fat high fructose-fed rats

Cardiac wall thickness

To study changes in the cardiac structure and function, we performed 2D echocardiography at the end of 12 and 20 weeks. At the end of 20 weeks, there was a significant increase in the intraventricular septum thickness (IVSd) and left ventricular posterior wall thickness (LVPWd) in the HFHFrD group, compared to the control group (Fig. 4A-B). HFHFrD $+\mathrm{P}$ and HFHFrD + E groups had significantly decreased IVSd and LVPWd compared to HFHFrD group, at the end of 20 weeks. Also, IVSd had significantly decreased in HFHFrD $+\mathrm{P}$ and HFHFrD $+\mathrm{E}$ groups between 12 and 20 weeks, indicating curative effect of the treatment with paricalcitol and enalapril (Fig. 4A-B). There was no significant difference between LVPWd of HFHFrD $+\mathrm{P}$ and HFHFHFrD $+\mathrm{E}$ groups between 12 and 20 weeks.

Cardiac chamber diameters

We did not observe significant changes in the diastolic left ventricular internal diameter (LVIDd) (Fig. $4 \mathrm{C}$ ). However, there was a significant increase in the systolic left ventricular internal diameter (LVIDs) of HFHFrD group compared to Con group, at the end of 20 weeks (Fig. 4D). This indicated dilatation of the left ventricle in HFHFrD group. HFHFrD $+\mathrm{P}$ and HFHFrD $+\mathrm{E}$ groups had significantly decreased LVIDs compared to HFHFrD group, at the end of 20 weeks. Also, LVIDs had significantly decreased in HFHFrD $+\mathrm{P}$ group between 12 and 20 weeks, indicating curative effect of the treatment with paricalcitol (Fig. 4D).

Cardiac contractile function

At the end of 20 weeks, there was a significant decrease in the fractional shortening (FS) and ejection fraction (EF) in the HFHFrD group, compared to the control group (Fig. 4E-F). While both HFHFrD+P and HFHFrD + E groups had significantly increased FS, only HFHFrD + P group had significantly increased EF compared to HFHFrD group, at the end of 20 weeks. Also, FS and EF had significantly increased in HFHFrD $+\mathrm{P}$ group between 12 and 20 weeks, indicating curative effect of the treatment with paricalcitol (Fig. 4E).

\section{Paricalcitol does not affect myocardial fibrosis in high fat high fructose-fed rats}

We observed no significant differences in histopathological features of different groups in haematoxylin eosin (H\&E)-stained rat heart sections (Fig. 5A). In the Masson trichrome stained heart sections, increased perivascular fibrosis was observed in HFHFrD group (Fig. 5B). However, no siginificant improvement was observed in HFHFrD $+\mathrm{P}$ and HFHFrD $+\mathrm{E}$ groups, compared to HFHFrD group (Fig. 5B).

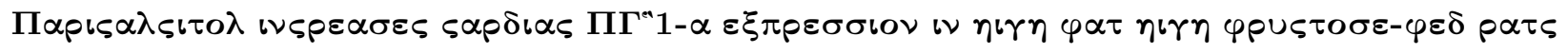
PGC1- $\alpha$ induces mitochondrial biogenesis through activation of transcription factors such as NRF-1 and NRF-2. At the end of the study, PGC1- $\alpha$ expression was lower in HFHFrD group, compared to Control group, though not statistically significant (Fig. 6B). Treatment with paricalcitol caused significantly higher expression of PGC1- $\alpha$ protein in HFHFrD $+\mathrm{P}$ group, compared to the HFHFrD group. The standard enalapril also significantly upregulated PGC1- $\alpha$ expression, similar to paricalcitol (Fig. 6B). 


\section{Paricalcitol does not alter mitochondrial mass/content in high fat high fructose-fed rats}

Since we observed increased expression of PGC1- $\alpha$ expression in the hearts of HFHFrD $+\mathrm{P}$ group, we evaluated the effect of paricalcitol on mitochondrial mass/content. In general, change in mitochondrial mass is directly correlated to the change in mitochondrial DNA content. We observed no significant differences in mitochondrial DNA/content between the different experimental groups, calculate from the expression of mitochondrial CO-1 normalised to beta 2-microglobin (Fig. 6D).

\section{Paricalcitol increases cardiac citrate synthase activity in high fat high fructose-fed rats}

At the end of the study, we evaluated citrate synthase activity in the rat hearts. It was significantly lower in HFHFrD group, compared to Con group (Fig. 6E). Treatment with paricalcitol, but not with enalapril, significantly increased citrate synthase activity in HFHFrD $+\mathrm{P}$ group, compared to HFHFrD group.

\section{Paricalcitol increases cardiac expression of Mfn2 in high fat high fructose-fed rats}

Since we observed increase in citrate synthase activity with paricalcitol treatment, we next studied the expression of Mfn1 and Mfn2 that are involved in mitochondrial fusion. There was no significant change in the expression of Mfn1 and Mfn2 in HFHFrD group compared to Con group (Fig. 7B). The increase in Mfn1 expression in both HFHFrD $+\mathrm{P}$ and HFHFrD $+\mathrm{E}$ groups was not significant, compared to HFHFrD group. However, paricalcitol and enalapril significantly increased the expression of Mfn2 in $\mathrm{HFHFrD}+\mathrm{P}$ group, compared to HFHFrD group (Fig. 7C).

\section{VDR knockdown causes downregulation of Mfn2 in H9c2 cardiomyoblasts}

Since paricalcitol upregulated the expression of Mfn2 in high fat high fructose-fed rat heart, we next investigated whether VDR is involved in the regulation of cardiac expression of mitofusins. We observed that VDR silencing in H9c2 cardiomyoblasts causes significant decrease in Mfn2 expression, but not in Mfn1 expression (Fig. 7E-F).

\section{DISCUSSION}

Vitamin D has been gaining attention for non-classical roles such as regulation of inflammation, immunity, cell cycle and metabolism. Association between vitamin D deficiency and adverse cardiometabolic outcomes has been repeatedly shown in patients, but the therapeutic role of vitamin D supplementation remains controversial [3]. Clinical studies have yielded conflicting results regarding the potential benefits of vitamin $\mathrm{D}$ in subjects with cardiometabolic diseases $[28,29]$. In view of reduced bioavailability of the active form of vitamin D in disease conditions, use of vitamin D analogues such as paricalcitol and maxacalcitol has been investigated [33]. Paricalcitol has shown therapeutic benefit in experimental models of kidney disease, diabetes and their cardiovascular complication [31,32]. However, its effect in context of metabolic syndromeassociated heart failure remained to be investigated. In this study, we have demonstrated the cardioprotective effects of paricalcitol treatment in high fat high fructose-fed rat model of metabolic syndrome.

Rats were fed on diets with high fat high fructose-diet to induce metabolic syndrome. Hypercaloric diets containing high fat and high fructose have been shown to induce hyperinsulinemia and impaired glucose tolerance [34]. Insulin resistance is a principal component of diet-induced metabolic syndrome. In our study, animals of the HFHFrD group showed elevated blood glucose, serum insulin and HOMA-IR values, indicating development of a prediabetes-like state. Treatment with paricalcitol for 8 weeks did not cause any significant changes in these parameters. However, paricalcitol reversed the impairment of blood glucose disposal in the intraperitoneal glucose tolerance test in $\mathrm{HFHFrD}+\mathrm{P}$ group.

High calorie diets are known to disturb serum lipid profile with an increase in serum triglycerides and free fatty acids [35]. In this study, we observed increase in triglycerides and free fatty acids in serum of rats fed of HFHFrD group. Treatment with paricalcitol attenuated these changes and improved lipid profile of the HFHFrD+P group. No significant differences were observed in the HDL-cholesterol levels of different groups. The lipid-lowering effect of paricalcitol in metabolic syndrome has not been reported earlier. 
Apart from triggering metabolic derangements such as insulin resistance and dyslipidemia, high fat high fructose diet also causes cardiovascular perturbations [34]. At the end of the study, we observed raised systolic, diastolic and mean arterial blood pressure in HFHFrD group. Treatment with paricalcitol significantly reduced blood pressure in the HFHFrD group. Hypertension is an established predictor of cardiac hypertrophy, due to resultant increase in cardiac workload. Heart weight-to-tail length ratio, an index of cardiac size, was increased significantly in the HFHFrD group compared to the Con group. Treatment with paricalcitol was associated with significantly decreased heart weight-to-tail length ratio in the HFHFrD+P group. Similar to our findings, paricalcitol has earlier been shown to attenuate cardiac hypertrophy in experimental models of pressure overload, hypertension and uremia [36, 37, 38].

As a consequence of its metabolic effects, high fat high fructose diet induces cardiac remodeling also [34]. At the end of 20 weeks, there was significant increase in the intraventricular septum and left ventricular posterior wall thicknesses in the HFHFrD rats. We observed significant decrease in cardiac wall thicknesses in the $\mathrm{HFHFrD}+\mathrm{P}$ group, indicating attenuation of cardiac hypertrophy. Though initially an adaptive response, unmitigated left ventricular hypertrophy progresses to cardiac failure. Metabolic syndrome is known to increase the risk of left ventricular systolic dysfunction by two folds [39]. In the HFHFrD rats, we observed increased systolic internal diameter of the left ventricle, indicating dilatation and systolic dysfunction. This led to significantly reduced fractional shortening and ejection fraction, which implies compromised cardiac contractility. In HFHFrD $+\mathrm{P}$ group, systolic function was markedly improved. According to earlier studies, paricalcitol prevents the progression of hypertrophy to heart failure in Dahl salt-sensitive hypertensive rats [37].

Interestingly, paricalcitol did not only attenuate the high fat high fructose-induced cardiac perturbations seen at the end of 20 weeks, but also reversed the cardiac dysfunction induced by high fat high fructose-diet at the end of 12 weeks. The improvement in systolic function is an interesting finding because the current treatment regimens for heart failure only manage the symptoms and do not reverse cardiac dysfunction. An important factor for inducing systolic dysfunction in high fat high fructose-fed rats is the fibrotic remodeling of the heart. Increased cardiac load due to factors such as hypertension, sympathetic tone and others can trigger reactive myocardial fibrosis [40]. These fibrotic changes usually begin in the perivascular region and slowly spread into the myocardium. Perivascular fibrosis was observed in the HFHFrD group through observation of Masson's trichrome-stained heart sections. We speculated that attenuation or reversal of this fibrosis might be responsible for cardioprotective effect of paricalcitol, seen earlier. However, we did not observe any significant difference in the HFHFrD $+\mathrm{P}$ group, as compared to the HFHFrD group. This is similar to earlier reports where paricalcitol had no protective effect on perivascular fibrosis in rats with renal insufficiency and low vitamin D [41]. These findings warranted further investigation into the factors underlying the protective effect of paricalcitol in high fat high fructose-induced cardiac dysfunction.

Since there were no significant improvements in cardiac structure or remodelling status, and functional improvements were observed, it indicated improvement in the functional status of the cardiac muscle. The myocardium relies on continuous supply of ATP to sustain its contractility. Mitochondria are the cellular organelles that generate the required ATP for energy transduction in heart. Defects in structure, function or dynamics of mitochondria adversely affect cardiac function. Mitochondrial dysfunction has been shown to underlie progression of cardiac hypertrophy to heart failure [46]. Hypercaloric diets can cause mitochondrial dysfunction through increased flux of fats and glucose [47]. Mitochondrial biogenesis, which implies formation of new mitochondria, might be impaired in conditions of unmitigated stress [48]. In our study also, we observed that expression of PGC1- $\alpha$ was downregulated in HFHFrD rat hearts. PGC1- $\alpha$ drives mitochondrial biogenesis through activation of downstream transcription factors [49]. In HFHFrD+P group, increased expression of PGC1- $\alpha$ was observed. In earlier reports, vitamin D supplementation has been shown to upregulate PGC1- $\alpha$ expression in rat skeletal muscle [50].

Increased cardiac expression of PGC1- $\alpha$ upon paricalcitol treatment led us to speculate an increase in mitochondrial mass/content in HFHFrD group. Mitochondrial mass/content can be quantified using mitochondria-tagging fluorescent dyes or by measuring the mitochondrial DNA content. We measured the 
expression of CO-1 gene that is encoded in mitochondrial DNA and normalised it to expression of beta 2-microbin encoded in nuclear DNA. Surprisingly, we did not observe any significant changes between the fold change of CO-1 expression between different groups. This indicated that there was no increase in mitochondrial biogenesis or number in $\mathrm{HFHFrD}+\mathrm{P}$ rat hearts, despite upregulation of PGC1- $\alpha$. However, activity of myocardial citrate synthase improved in HFHFrD $+\mathrm{P}$ group, compared to HFHFrD group. Acetyl CoA generated from glycolysis and fatty acid oxidation enters Kreb's cycle in the mitochondrial matrix. Citrate synthase is the first enzyme of this biochemical cycle and its activity is thus considered a hallmark of mitochondrial health [51]. Our findings indicate that paricalcitol improves mitochondrial function in high fat high fructose-fed rats.

Our next question was how mitochondrial function could improve without any changes in mitochondrial content or biogenesis. Mitochondrial dynamics are controlled not only through biogenesis but also through fusion and fission. Mitochondrial fusion improves mitochondrial function by fusing of mitochondria and recycling of damaged components [267]. We examined the expression of mitofusins (Mfn2) that are the proteins involved in mitochondrial fusion. Myocardial expression of Mfn2 increased significantly in $\mathrm{HFHFrD}+\mathrm{P}$ group, compared to HFHFrD group. Mfn2 has been earlier described as a therapeutic target for diabetic cardiomyopathy through inhibition of mitochondrial fission in $\mathrm{db} / \mathrm{db}$ mice [53]. It has also been shown to be protective against angiotensin-mediated cardiomyocyte injury by induction of mitophagy and mitochondrial fusion [52].

From the above findings, we could conclude that paricalcitol improved mitochondrial function possibly through improvement of mitochondrial dynamics. To confirm that this beneficial effect was mediated by activation of VDR by paricalcitol, we performed further experiments in vitro. To perform loss-of-function studies regarding the effect of VDR on mitochondrial fusion, we silenced VDR in H9c2 cardiomyoblasts. We observed that the expression of Mfn2 was remarkably reduced in H9c2 cells with VDR knockdown. This finding provided conclusive evidence about the positive role of VDR in mitochondrial dynamics.

In this study, we demonstrate that paricalcitol, a vitamin $\mathrm{D}$ receptor activator attenuates metabolic syndrome-associated cardiac dysfuncin rats. High fat high fructose diet induces glucose intolerance, dyslipidemia, hypertension, fibrosis and cardiac dysfunction in rats. Paricalcitol improves glucose tolerance, lipid profile, blood pressure and cardiac contractility, but not myocardial fibrosis. We demonstrate that the VDR activator paricalcitol improves cardiometabolic function in rats possibly through mitochondrial reprogramming. Despite increase in cardiac PGC1- $\alpha$ expression, mitochondrial biogenesis is not observed in paricalcitol-treated rats with metabolic syndrome. However, increase in expression of Mfn2 indicates the role of VDR activation in mitofusion. These findings indicate potential for repurposing of paricalcitol for treatment of cardiac failure associated with conditions such as prediabetes and diabetes mellitus.

\section{DECLARATIONS}

\section{Acknowledgments}

THSTI core funds supported this work. The authors wish to acknowledge CSIR and ICMR for providing Senior Research Fellowships to HLN and PBK, respectively.

\section{Author Contributions}

HLN and PBK carried out animal experiments, molecular and biochemical estimation, and statistical analysis of results. PP performed echocardiographic examination. RA and SS provided assistance for animal experiments. SA performed histopathological examination. PC and SKM supervised and coordinated the echocardiography. HLN and SKB participated in conception and design of the study, data interpretation, and preparation of the manuscript.

\section{Conflict of Interest statement}

The authors declare that they have no conflict of financial or non-financial interests.

\section{References}


1. Conrad N, Judge A, Tran J, Mohseni H, Hedgecott D, Crespillo AP, Allison M, Hemingway H, Cleland JG, McMurray JJ, Rahimi K: Temporal trends and patterns in heart failure incidence: a populationbased study of 4 million individuals. The Lancet 2018; 391(10120):572-580.

2. McMurray JJ: Systolic heart failure. New England Journal of Medicine 2010; 362(3):228-30.

3. Adela R, Borkar RM, Bhandi MM, Vishwakarma G, Reddy PNC, Srinivas R, Banerjee SK: Lower vitamin D metabolites levels were associated with increased coronary artery diseases in type 2 diabetes patients in India. Scientific reports 2016; 6:37593.

4. Rostand SG and Drüeke TB: Parathyroid hormone, vitamin D, and cardiovascular disease in chronic renal failure. Kidney international 1999;56(2):383-392.

5. Simpson RU, Hershey SH, Nibbelink KA: Characterization of heart size and blood pressure in the vitamin D receptor knockout mouse. The Journal of steroid biochemistry and molecular biology 2007;103(3-5): 521-524

6. Weishaar RE, Kim SN, Saunders DE, Simpson RU: Involvement of vitamin D3 with cardiovascular function. III. Effects on physical and morphological properties. American Journal of PhysiologyEndocrinology And Metabolism 1990;43(1):E134-E142.

7. Sugden JA, Davies JI, Witham MD, Morris AD, Struthers AD: Vitamin D improves endothelial function in patients with Type 2 diabetes mellitus and low vitamin D levels. Diabetic medicine 2008;25(3):320325 .

8. Weishaar RE, Kim SN, Saunders DE, Simpson RU: Involvement of vitamin D3 with cardiovascular function. III. Effects on physical and morphological properties. American Journal of PhysiologyEndocrinology And Metabolism 1990;43(1): E134-E142.

9. Musso G, Cassader M, Cohney S, De Michieli F, Pinach S, Saba F, Gambino R: Fatty liver and chronic kidney disease: novel mechanistic insights and therapeutic opportunities. Diabetes care 2016;39(10):1830-1845.

10. Wortsman J, Matsuoka LY, Chen TC, Lu Z, Holick MF: Decreased bioavailability of vitamin D in obesity. The American journal of clinical nutrition 2000;72(3):690-693.

11. Bae S, Yalamarti B, Ke Q, Choudhury S, Yu H, Karumanchi SA, Kroeger P, Thadhani R, Kang PM: Preventing progression of cardiac hypertrophy and development of heart failure by paricalcitol therapy in rats. Cardiovascular research 2011;91(4):632-639.

12. Zoccali C, Curatola G, Panuccio V, Tripepi R, Pizzini P, Versace M, Bolignano D, Cutrupi S, Politi R, Tripepi G, Ghiadoni L: Paricalcitol and endothelial function in chronic kidney disease trial. Hypertension, 2014;64(5):1005-1011.

13. Sorokina N, O'Donnell JM, McKinney RD, Pound KM, Woldegiorgis G, LaNoue KF, Ballal K, Taegtmeyer H, Buttrick PM, Lewandowski ED: Recruitment of compensatory pathways to sustain oxidative flux with reduced CPT1 activity characterizes inefficiency in energy metabolism in hypertrophied hearts. Circulation 2007;115(15):2033-2041.

14. Parra V, Verdejo HE, Iglewski M, Del Campo A, Troncoso R, Jones D, Zhu Y, Kuzmicic J, Pennanen C, Lopez-Crisosto C, Jana F: Insulin stimulates mitochondrial fusion and function in cardiomyocytes via the Akt-mTOR-NFxB-Opa-1 signaling pathway. Diabetes 2014;63(1):75-88.

15. Zhang L, Jaswal JS, Ussher JR, Sankaralingam S, Wagg C, Zaugg M, Lopaschuk GD: Cardiac insulinresistance and decreased mitochondrial energy production precede the development of systolic heart failure after pressure-overload hypertrophy. Circulation: Heart Failure 2013;6(5):1039-1048.

16. Zhou X, Chen J: Is treatment with trimetazidine beneficial in patients with chronic heart failure?. PLoS One 2014;9(5):e94660.

17. Lopatin Y. Metabolic therapy in heart failure. Cardiac failure review 2015;1(2):112.

18. Calton EK, Keane KN, Soares MJ: The potential regulatory role of vitamin D in the bioenergetics of inflammation. Current Opinion in Clinical Nutrition \& Metabolic Care 2015;18(4):367-373.

19. Adela R, Borkar RM, Mishra N, Bhandi MM, Vishwakarma G, Varma BA, Ragampeta S, Banerjee SK: Lower serum vitamin D metabolite levels in relation to circulating cytokines/chemokines and metabolic hormones in pregnant women with hypertensive disorders. Frontiers in immunology 2017;8:273.

20. Manna P, Achari AE, Jain SK: 1, $25(\mathrm{OH})$ 2-vitamin D 3 upregulates glucose uptake mediated by 
SIRT1/IRS1/GLUT4 signaling cascade in C2C12 myotubes. Molecular and cellular biochemistry 2018;444(1-2):103-108.

21. Marcotorchino J, Tourniaire F, Astier J, Karkeni E, Canault M, Amiot MJ, Bendahan D, Bernard M, Martin JC, Giannesini B, Landrier JF: Vitamin D protects against diet-induced obesity by enhancing fatty acid oxidation. The Journal of nutritional biochemistry 2014;25(10):1077-1083.

22. Asemi Z, Hashemi T, Karamali M, Samimi M, Esmaillzadeh A: Effects of vitamin D supplementation on glucose metabolism, lipid concentrations, inflammation, and oxidative stress in gestational diabetes: a double-blind randomized controlled clinical trial. The American journal of clinical nutrition 2013;98(6):1425-1432.

23. Calton EK, Keane KN, Soares MJ, Rowlands J, Newsholme P: Prevailing vitamin D status influences mitochondrial and glycolytic bioenergetics in peripheral blood mononuclear cells obtained from adults. Redox biology 2016;10:243-250.

24. Peng X, Shang G, Wang W, Chen X, Lou Q, Zhai G, Li D, Du Z, Ye Y, Jin X, He J: Fatty acid oxidation in zebrafish adipose tissue is promoted by $1 \alpha, 25(\mathrm{OH})$ 2D3. Cell reports 2017;19(7):1444-1455.

25. Alkharfy KM, Al-Daghri NM, Ahmed M, Yakout SM: Effects of vitamin D treatment on skeletal muscle histology and ultrastructural changes in a rodent model. Molecules 2012;17(8):9081-9089.

26. Ricca C, Aillon A, Bergandi L, Alotto D, Castagnoli C, Silvagno F: Vitamin D receptor is necessary for mitochondrial function and cell health. International journal of molecular sciences 2018;19(6):1672.

27. Longoni A, Kolling J, Siebert C, dos Santos JP, da Silva JS, Pettenuzzo LF, Meira-Martins LA, Gonçalves CA, de Assis AM, Wyse AT: 1, 25-Dihydroxyvitamin D3 prevents deleterious effects of homocysteine on mitochondrial function and redox status in heart slices. Nutrition Research 2017;38:5263.

28. Zhang, Q., Cheng, Y., He, M., Li, T., Ma, Z., \& Cheng, H. (2016).Effect of various doses of vitamin D supplementation on pregnant women with gestational diabetes mellitus: a randomized controlled trial.Experimental and therapeutic medicine, 12(3), 1889-1895.

29. Swart, K. M., Lips, P., Brouwer, I. A., Jorde, R., Heymans, M. W., Grimnes, G., et al. (2018). Effects of vitamin $\mathrm{D}$ supplementation on markers for cardiovascular disease and type 2 diabetes: an individual participant data meta-analysis of randomized controlled trials. The American journal of clinical nutrition, 107(6), 1043-1053.

30. Parsanathan, R., \& Jain, S. K. (2018). Glutathione Deficiency Induces Epigenetic Alterations of Vitamin D Metabolism Genes in the Liver of High-Fat Diet-Induced Type 2 Diabetic Mice.

31. Lai, C. C., Liu, C. P., Cheng, P. W., Lu, P. J., Hsiao, M., Lu, W. H., et al. (2016). Paricalcitol attenuates cardiac fibrosis and expression of endothelial cell transition markers in isoproterenol-induced cardiomyopathic rats. Critical care medicine, 44(9), e866-e874.

32. Yildirim, Y., YILMAZ, Z., Kadiroglu, A. K., Kara, A. V., Bahadir, V., Aydin, E., et al. (2016). Mp179 pretreatment with paricalcitol attenuates oxidative stress in renal ischemia reperfusion induced nephropaty in rats.

33. Wu-Wong, J. R. (2009). Potential for vitamin D receptor agonists in the treatment of cardiovascular disease. British journal of pharmacology, 158(2), 395-412.

34. Panchal, S. K., Poudyal, H., Iyer, A., Nazer, R., Alam, A., Diwan, V., et al. (2011). High-carbohydrate high-fat diet-induced metabolic syndrome and cardiovascular remodeling in rats.Journal of cardiovascular pharmacology, 57(1), 51-64.

35. Stranahan, A. M., Norman, E. D., Lee, K., Cutler, R. G., Telljohann, R. S., Egan, J. M., \& Mattson, M. P. (2008). Diet-induced insulin resistance impairs hippocampal synaptic plasticity and cognition in middle-aged rats. Hippocampus, 18(11), 1085-1088.

36. Meems, L. M., Cannon, M. V., Mahmud, H., Voors, A. A., van Gilst, W. H., Sillje, H. H., et al. (2012). The vitamin D receptor activator paricalcitol prevents fibrosis and diastolic dysfunction in a murine model of pressure overload. The Journal of steroid biochemistry and molecular biology, 132(3-5), 282-289.

37. Bae, S., Yalamarti, B., Ke, Q., Choudhury, S., Yu, H., Karumanchi, S. A., et al, P. M. (2011). Preventing progression of cardiac hypertrophy and development of heart failure by paricalcitol therapy 
in rats. Cardiovascular research, 91(4), 632-639.

38. Freundlich, M., Li, Y. C., Quiroz, Y., Bravo, Y., Seeherunvong, W., Faul, C., et al. (2013). Paricalcitol downregulates myocardial renin-angiotensin and fibroblast growth factor expression and attenuates cardiac hypertrophy in uremic rats. American journal of hypertension, 27(5), 720-726.

39. Gong, H. P., Tan, H. W., Fang, N. N., Song, T., Li, S. H., Zhong, M., et al. (2009). Impaired left ventricular systolic and diastolic function in patients with metabolic syndrome as assessed by strain and strain rate imaging. Diabetes research and clinical practice, 83(3), 300-307.

40. Wu, H., Chen, L., Xie, J., Li, R., Li, G. N., Chen, Q. H., et al. (2016). Periostin expression induced by oxidative stress contributes to myocardial fibrosis in a rat model of high salt-induced hypertension. Molecular medicine reports, 14(1), 776-782.

41. Repo, J. M., Rantala, I. S., Honkanen, T. T., Mustonen, J. T., Koobi, P., Tahvanainen, A. M., et al. (2007). Paricalcitol aggravates perivascular fibrosis in rats with renal insufficiency and low calcitriol. Kidney international, 72(8), 977-984.

42. Ross EA, Tian J, Abboud H, Hippensteel R, Melnick JZ, et al. (2008) Oral paricalcitol for the treatment of secondary hyperparathyroidism in patients on hemodialysis or peritoneal dialysis. American journal of nephrology 28: 97-106.

43. Thethi TK, Bajwa MA, Ghanim H, Jo C, Weir M, et al. (2015) Effect of paricalcitol on endothelial function and inflammation in type 2 diabetes and chronic kidney disease. Journal of Diabetes and its Complications 29: 433-437.

44. Bae S, Yalamarti B, Ke Q, Choudhury S, Yu H, et al. (2011) Preventing progression of cardiac hypertrophy and development of heart failure by paricalcitol therapy in rats. Cardiovascular research 91: $632-639$.

45. Hu X, Shang J, Yuan W, Zhang S, Jiang Y, et al. (2018) Effects of paricalcitol on cardiovascular outcomes and renal function in patients with chronic kidney disease. Herz 43: 518-528.

46. Rosca MG, Tandler B, Hoppel CL (2013) Mitochondria in cardiac hypertrophy and heart failure. Journal of molecular and cellular cardiology 55: 31-41.

47. Jelenik T, Roden M (2013) Mitochondrial plasticity in obesity and diabetes mellitus. Antioxidants \& redox signaling 19: 43-268.

48. Sivitz WI, Yorek MA (2010) Mitochondrial dysfunction in diabetes: from molecular mechanisms to functional significance and therapeutic opportunities. Antioxidants \& redox signaling 12: 537-577.

49. Scarpulla RC (2011) Metabolic control of mitochondrial biogenesis through the PGC-1 family regulatory network. Biochimica et Biophysica Acta (BBA)-Molecular Cell Research 1813: 152-1278.

50. Savkur RS, Bramlett KS, Stayrook KR, Nagpal S, Burris TP (2005) Coactivation of the human vitamin $D$ receptor by the peroxisome proliferator-activated receptor $\gamma$ coactivator-1 $\alpha$. Molecular pharmacology 68: 511-517.

51. REMINGTON SJ (1992) Structure and mechanism of citrate synthase. Current topics in cellular regulation: Elsevier. pp. 209-229.

52. Meyer JN, Leuthner TC, Luz AL (2017) Mitochondrial fusion, fission, and mitochondrial toxicity. Toxicology 391: 42-53.

53. Hu L, Ding M, Tang D, Gao E, Li C, et al. (2019) Targeting mitochondrial dynamics by regulating Mfn2 for therapeutic intervention in diabetic cardiomyopathy. Theranostics 9: 3687.

\section{Figure Legends:}

Fig. 1 Schematic representation of experimental animal study design

Fig. 2 Paricalcitol improves glucose tolerance in high fat high fructose-fed rats. A. Fasting blood glucose B. Fasting serum insulin C. HOMA IR D. HOMA-B E.Intraperitoneal glucose tolerance test (12 weeks) F.Intraperitoneal glucose tolerance test (20 weeks) G. Area Under Curve (20 weeks IPGTT). Data expressed as mean \pm SEM $(\mathrm{n}=6) .{ }^{*} \mathrm{p}<0.05,{ }^{* *} \mathrm{p}<0.01,{ }^{* * *} \mathrm{p}<0.001$ vs. Con group. ${ }^{\#} \mathrm{p}<0.05$ vs. HFHFrD group.

Fig. 3 Paricalcitol attenuates dyslipdemia, hypertension and cardiac hypertrophy in high fat 
high fructose-fed rats. A. Serum triflycerides B. Serum free fatty acids C.Serum HDL cholesterol D. Systolic blood pressure E.Diastolic blood pressure F. Mean arterial pressure G.Heart weight/Tail length ratio. Data expressed as mean \pm SEM $(\mathrm{n}=6) .{ }^{*} \mathrm{p}<0.05,{ }^{* *} \mathrm{p}<0.01$ vs. CON group, ${ }^{\#} \mathrm{p}<0.05,{ }^{\# \#} \mathrm{p}<0.01,{ }^{\# \# \#} \mathrm{p}<0.001$ vs. HFHFrD group.

Fig. 4 Paricalcitol reverses cardiac hypertrophy and failure in high fat high fructose-fed rats. A. Intraventricular septum thickness (diastole) B. Left ventricular posterior wall thickness (diastole) C. Left ventricular internal dimension (diastolic)D. Left ventricular internal dimension (systolic) E.Fractional shortening F. Ejection fraction. Data expressed as mean \pm SEM $(\mathrm{n}=4-5) .{ }^{*} \mathrm{p}<0.05,{ }^{* *} \mathrm{p}<0.01{ }^{* * *} \mathrm{p}<0.001$ vs. CON group at 20 weeks. ${ }^{\#} \mathrm{p}<0.05,{ }^{\# \# \#} \mathrm{p}<0.001$ vs. HFHFrD group at 20 weeks. ${ }^{@} \mathrm{p}<0.05,{ }^{@} \mathrm{p}<0.01$ vs. same group at 12 weeks.

Fig. 5 Paricalcitol does not affect myocardial fibrosis in high fat high fructose-fed rats. A. Representative micrograph of haematoxylin-eosin stained rat heart sections B. Representative micrograph of Masson's trichrome- stained rat heart sections.

Fig. 6 Paricalcitol does not alter myocardial mitochondrial content in high fat high fructose-fed rats. A.Representative western blot image of PGC1- $\alpha$ B. Densitometry analysis of PGC1- $\alpha$ expression C. Representative agarose gel image of CO-1 and beta 2-microglobin PCR products D.Densitometry analysis of CO-1 expression. E. Fold change in citrate synthase activity. Protein expression data were normalised to the expression of stain free gel (TGX Stain-FreeTM FastCastTM BioRadTM) image. \#\# $\mathrm{p}<0.01$ vs. HFHFrD group. CO-1 expression data were normalised to beta 2-microglobin expression. Data expressed as mean \pm SEM ( $\mathrm{n}=3$ for Western blot and agarose gel electrophoresis; $\mathrm{n}=5$ for citrate synthase activity). ${ }^{*} \mathrm{p}<0.05$ vs. Control, $\# \# \mathrm{p}<0.01$ vs. HFHFrD.

Fig. 7 Vitamin D receptor modulates myocardial expression of mitofusin 2 A. Representative western blot images of Mfn1 and Mfn2 in rat hearts. B. Densitometry analysis of Mfn1 expression in rat hearts C. Densitometry analysis of Mfn2 expression in rat hearts. D. Representative western blot images of Mfn1 and Mfn 2 in H9c2 cells E. Densitometry analysis of Mfn1 in H9c2 cellsF. Densitometry analysis of Mfn2 in H9c2 cells. Protein expression data were normalised to the expression of stain free gel (TGX StainFreeTM FastCastTM BioRadTM) image or to the expression of beta actin. Data expressed as mean \pm SEM $(\mathrm{n}=3) .{ }^{*} \mathrm{p}<0.05$ vs. Control group, ${ }^{* * *} \mathrm{p}<0.001$ vs. Control group.

\section{Hosted file}

Figure 1.pptx available at https://authorea.com/users/330850/articles/457670-paricalcitolreverses-metabolic-syndrome-associated-heart-failure-through-enhanced-mitochondrialfusion

\section{Hosted file}

Figure 2.pptx available at https://authorea.com/users/330850/articles/457670-paricalcitolreverses-metabolic-syndrome-associated-heart-failure-through-enhanced-mitochondrialfusion

\section{Hosted file}

Figure 3.pptx available at https://authorea.com/users/330850/articles/457670-paricalcitolreverses-metabolic-syndrome-associated-heart-failure-through-enhanced-mitochondrialfusion

\section{Hosted file}

Figure 4.pptx available at https://authorea.com/users/330850/articles/457670-paricalcitolreverses-metabolic-syndrome-associated-heart-failure-through-enhanced-mitochondrialfusion

\section{Hosted file}


Figure 5.pptx available at https://authorea.com/users/330850/articles/457670-paricalcitolreverses-metabolic-syndrome-associated-heart-failure-through-enhanced-mitochondrialfusion

\section{Hosted file}

Figure 6.pptx available at https://authorea.com/users/330850/articles/457670-paricalcitolreverses-metabolic-syndrome-associated-heart-failure-through-enhanced-mitochondrialfusion

\section{Hosted file}

Figure 7.pptx available at https://authorea.com/users/330850/articles/457670-paricalcitolreverses-metabolic-syndrome-associated-heart-failure-through-enhanced-mitochondrialfusion

\section{Hosted file}

Table 1.docx available at https://authorea.com/users/330850/articles/457670-paricalcitolreverses-metabolic-syndrome-associated-heart-failure-through-enhanced-mitochondrialfusion 